\title{
Smac/DIABLO expression in human gastrointestinal carcinoma: Association with clinicopathological parameters and survivin expression
}

\author{
MICHIKO SHINTANI $^{1}$, AKIKO SANGAWA ${ }^{1,2}$, NAOKI YAMAO ${ }^{3}$ and SHINGO KAMOSHIDA ${ }^{1}$ \\ ${ }^{1}$ Laboratory of Pathology, Division of Medical Biophysics, Kobe University Graduate School of Health Sciences, \\ Kobe, Hyogo 654-0142; ${ }^{2}$ Department of Diagnostic Pathology, Osaka Red Cross Hospital, Osaka 543-8555; \\ ${ }^{3}$ Department of Clinical Laboratory, Kuma Hospital, Kobe, Hyogo 650-0011, Japan
}

Received February 25, 2014; Accepted September 8, 2014

DOI: $10.3892 / 01.2014 .2598$

\begin{abstract}
Lack of apoptosis is a key factor in carcinogenesis and tumor progression. Survivin is a member of the inhibitor of apoptosis protein (IAP) family. Second mitochondria-derived activator of caspases/direct inhibitor of apoptosis-binding protein with low $\mathrm{pI}$ (Smac/DIABLO) is an antagonist of IAPs. Recently, Smac/DIABLO was identified as a potent therapeutic target. However, the clinical significance of Smac/DIABLO in gastrointestinal carcinomas remains unclear. In the present study, Smac/DIABLO expression was analyzed by immunohistochemistry in 72 gastric adenocarcinomas and 78 colorectal adenocarcinomas. The expression of Smac/DIABLO was significantly higher in colorectal carcinoma than in gastric carcinoma. Additionally, a correlation was found between the expression of Smac/DIABLO and nuclear survivin in well- to moderately-differentiated colorectal adenocarcinomas $(\mathrm{r}=0.245 ; \mathrm{P}<0.01)$. Based on these results, it was hypothesized that gastric and colorectal carcinomas differ in the level of Smac/DIABLO expression. Our previous studies revealed that the expression of cleaved caspase- 9 was significantly lower in colorectal carcinoma than in gastric carcinoma $(\mathrm{P}<0.0001)$. Conversely, the expression levels of microtubule-associated protein 1 light chain 3 (LC3), an autophagy marker, and survivin were significantly higher in colon cancer than in gastric cancer $(\mathrm{P}<0.0001$ and $\mathrm{P}<0.01$, respectively). Taken together, these results indicate that not only LC3 and survivin expression, but also Smac/DIABLO expression, are significantly higher in colorectal carcinoma than in gastric carcinoma. We hypothesize that the analysis
\end{abstract}

Correspondence to: Dr Michiko Shintani, Laboratory of Pathology, Division of Medical Biophysics, Kobe University Graduate School of Health Sciences, 7-10-2 Tomogaoka, Kobe, Hyogo 654-0142, Japan

E-mail: mtshin@kobe-u.ac.jp

Key words: survivin, Smac/DIABLO, immunohistochemistry, apoptosis of Smac/DIABLO, survivin and LC3 expression in colorectal carcinoma is likely to aid cancer therapy due to the involvement of these markers in apoptosis and/or autophagy.

\section{Introduction}

Second mitochondria-derived activator of caspases/direct inhibitor of apoptosis-binding protein with low pI (Smac/DIABLO) is a mitochondrial apoptogenic molecule that is released from the mitochondria in response to apoptotic stress. Smac/DIABLO is known to antagonize the function of inhibitors of apoptosis proteins (IAPs), including X-linked inhibitor of apoptosis (XIAP), cellular IAP1/2 and survivin (1-7). The lack of Smac/DIABLO expression may inhibit apoptosis in cancer cells, promoting survival. Previous studies have indicated that decreased levels of Smac/DIABLO correlate with cancer progression. Smac/DIABLO is considered a potent therapeutic target (8-11). However, the clinical significance of Smac/DIABLO in various cancers remains unclear.

Survivin, a member of the IAP family, exhibits a dual cellular function as an inhibitor of apoptosis and a regulator of mitosis. The anti-apoptotic effect of survivin is associated with the inhibition of caspase activity. Furthermore, survivin also acts as a chromosome passenger protein, regulating the $\mathrm{G}_{2}$ and $\mathrm{M}$ phases of the cell cycle (12-17). High levels of survivin expression are found in numerous embryonic tissues and in the majority of human tumors. By contrast, extremely low level or undetectable levels of expression are found in differentiated adult tissues. As a result, survivin may prove to be a marker for tumor progression and prognosis (18-20).

Apoptosis is a primary biochemical cell-death pathway that is critical for normal tissue homeostasis, cellular differentiation and development $(21,22)$. Apoptosis is regulated by the activity of caspases via two possible pathways, the death receptor-mediated apoptotic pathway (extrinsic pathway, involving caspase 8) and the mitochondrial-mediated apoptotic pathway (intrinsic pathway, involving caspase 9) (21-23). Generally, IAPs suppress apoptosis via the inhibition of caspase activation.

The expression of cleaved caspase (CC) 8 and 9, microtubule-associated protein 1 light chain 3 (LC3), an autophagy 
marker, and survivin in gastric and colorectal carcinomas has been investigated to elucidate the cell death pathway $(24,25)$. Results from our previous studies revealed that the expression of CC9 in colorectal carcinoma was significantly lower than that in gastric carcinoma $(\mathrm{P}<0.0001)(24)$. By contrast, the expression of LC3 and survivin in colon carcinoma was significantly higher than that in gastric carcinoma $(\mathrm{P}<0.0001$ and $\mathrm{P}<0.01$, respectively) $(24,25)$. These results indicated that different cellular death pathways are activated in gastric and colorectal carcinomas (24).

In the present study, the expression of Smac/DIABLO in gastric and colorectal carcinoma was examined using immunohistochemistry. Furthermore, the association between Smac/DIABLO expression and clinicopathological parameters was investigated. Additionally, the correlation between the expression of Smac/DIABLO and survivin was elucidated.

\section{Materials and methods}

Tissue samples. Surgically resected tumor tissues were collected from the archives of the Department of Diagnostic Pathology of the Osaka Red Cross Hospital (Osaka, Japan) and the Kobe Central Hospital of Social Insurance (Kobe, Japan). A total of 72 advanced gastric adenocarcinomas (36 well- to moderately-differentiated and 36 poorly-differentiated) and 78 colorectal adenocarcinomas (68 well- to moderately-differentiated and 10 poorly-differentiated) were analyzed for Smac/DIABLO expression by immunohistochemistry. The study was approved by the ethics committee of Kobe University Graduate School of Health Sciences (Kobe, Japan). The tumors were classified according to the tumor-node-metastasis (TNM) classification of malignant tumors (TNM 2009) (26). All specimens were preserved in $10 \%$ formalin and embedded in paraffin. Sections that were $3-\mu \mathrm{m}$ thick were cut consecutively and mounted on aminopropyltriethoxysilane-coated slides.

Immunohistochemical staining. Smac/DIABLO expression was analyzed using immunohistochemistry. The tissue sections were deparaffinized with xylene (Nacalai Tesque, Inc., Kyoto, Japan) and dehydrated using a graded series of ethanol solutions. Antigen retrieval was performed by immersing the slides in $10 \mathrm{mM}$ citrate buffer (pH 7.0; Nacalai Tesque, Inc.) and heating for $10 \mathrm{~min}$ in a pressure cooker (Tefal, Haute-Savoie, France). The sections were then cooled at room temperature in a soaking solution (10 mM citrate buffer; $\mathrm{pH} 7.0$; Nacalai Tesque, Inc.) for $30 \mathrm{~min}$. Next, the sections were washed with water, followed by $10 \mathrm{mM}$ phosphate-buffered saline (PBS; $\mathrm{pH}$ 7.2). Following blocking with $0.25 \%$ casein in PBS (Dako, Glostrup, Denmark), the sections were incubated with a mouse monoclonal antibody against Smac/DIABLO (1:1,000; Cell Signaling Technology, Inc., Danvers, USA) overnight at room temperature. The sections were then rinsed with PBS. To detect Smac/DIABLO, the sections were incubated with Histofine Simple Stain MAX-PO (Nichirei Bioscience Inc., Tokyo, Japan) for $1 \mathrm{~h}$ at room temperature. The reaction products were then developed using 3,3'-diaminobenzidine (Dako, Glostrup, Denmark) and counterstained using Mayer's hematoxylin (Merck KGaA, Darmstadt, Germany). For the negative control, a section was treated as described above, but PBS was used instead of the primary antibody.
Evaluation of immunostaining. Sections were considered positive for Smac/DIABLO if cytoplasmic staining was observed. A mean percentage of positive tumor cells was determined in $\geq 5$ areas at a magnification of $x 400$ and assigned to the following categories: 0 , negative; $1,<30 \% ; 2,30-69 \%$; and $3, \geq 70 \%$. The intensity of Smac/DIABLO immunostaining was scored as follows: 1 , weak; 2 , moderate; and 3 , intense. A final score was obtained by calculating the sum of these two scores. Cases with final scores of $<5$ were considered to exhibit low expression, whereas cases with final scores of $\geq 5$ were considered to exhibit high expression.

Statistical analysis. Differences in the expression of Smac/DIABLObetween gastric and colorectaladenocarcinomas, well- to moderately-differentiated and poorly-differentiated adenocarcinomas, patient age and gender, as well as lymphatic and vascular invasion were evaluated using the $\chi^{2}$ and Fisher's exact tests. The correlation between marker expression and tumor location, depth of invasion, lymph node metastasis and pathological stage were analyzed using the Kruskal-Wallis test. Spearman's rank correlation was used to assess the correlation between the different markers. $\mathrm{P}<0.05$ was considered to indicate a statistically significant difference.

\section{Results}

Expression of Smac/DIABLO in gastric and colorectal carcinomas. Smac/DIABLO expression was predominantly observed in the cytoplasm (Fig. 1). Smac/DIABLO-positive staining was observed in $46 \%$ (33/72) of gastric carcinomas. Smac/DIABLO expression was higher in well- to moderately-differentiated specimens $(50 \%)$ compared with poorly-differentiated specimens (42\%), however, no significant differences were identified (Table I).

Smac/DIABLO-positive staining. Smac/DIABLO-positive staining was observed in $69 \%(54 / 78)$ of colorectal carcinomas. The expression of Smac/DIABLO was significantly higher in colorectal carcinoma than in gastric carcinoma $(\mathrm{P}<0.01)$. Smac/DIABLO expression was higher in well- to moderately-differentiated specimens (71\%) compared with poorly-differentiated specimens $(60 \%)$, however, no significant differences were identified.

Correlation between Smac/DIABLO expression and clinicopathological parameters. Tables II and III present the associations between Smac/DIABLO protein expression and the clinicopathological parameters. In gastric carcinomas, none of the parameters of patient age, gender, tumor location, depth of invasion, lymph-node metastasis, lymphatic invasion, vascular invasion or pathological stage were found to be associated with the positive expression of Smac/DIABLO. In colorectal carcinomas, the level of Smac/DIABLO expression was significantly higher in cases without vascular invasion than in the cases with vascular invasion $(\mathrm{P}<0.05)$. However, none of the other parameters of patient age, gender, tumor location, depth of invasion, lymph-node metastasis, lymphatic invasion, or pathological stage were found to be associated with positive Smac/DIABLO expression. Although no significant difference was identified between the majority of the clinicopathological 
Table I. Expression of Smac/DIABLO in gastrointestinal adenocarcinomas.

\begin{tabular}{lccc}
\hline Adenocarcinoma type & Total, $\mathrm{n}$ & High expression, $\mathrm{n}(\%)$ & Low expression, $\mathrm{n}(\%)$ \\
\hline Gastric adenocarcinoma & 72 & $33(46)^{\mathrm{a}}$ & $39(54)$ \\
Well- to moderately-differentiated & 36 & $18(50)$ & $18(50)$ \\
Poorly-differentiated & 36 & $15(42)$ & $21(58)$ \\
Colorectal adenocarcinoma & 78 & $54(69)^{\mathrm{a}}$ & $24(31)$ \\
Well- to moderately-differentiated & 68 & $48(71)$ & $20(29)$ \\
Poorly-differentiated & 10 & $6(60)$ & $4(40)$ \\
\hline
\end{tabular}

${ }^{a} \mathrm{P}<0.01$, gastric adenocarcinoma vs. colotrectal adenocarcinoma. Smac/DIABLO, second mitochondria-derived activator of caspases/direct inhibitor of apoptosis-binding protein with low pI.
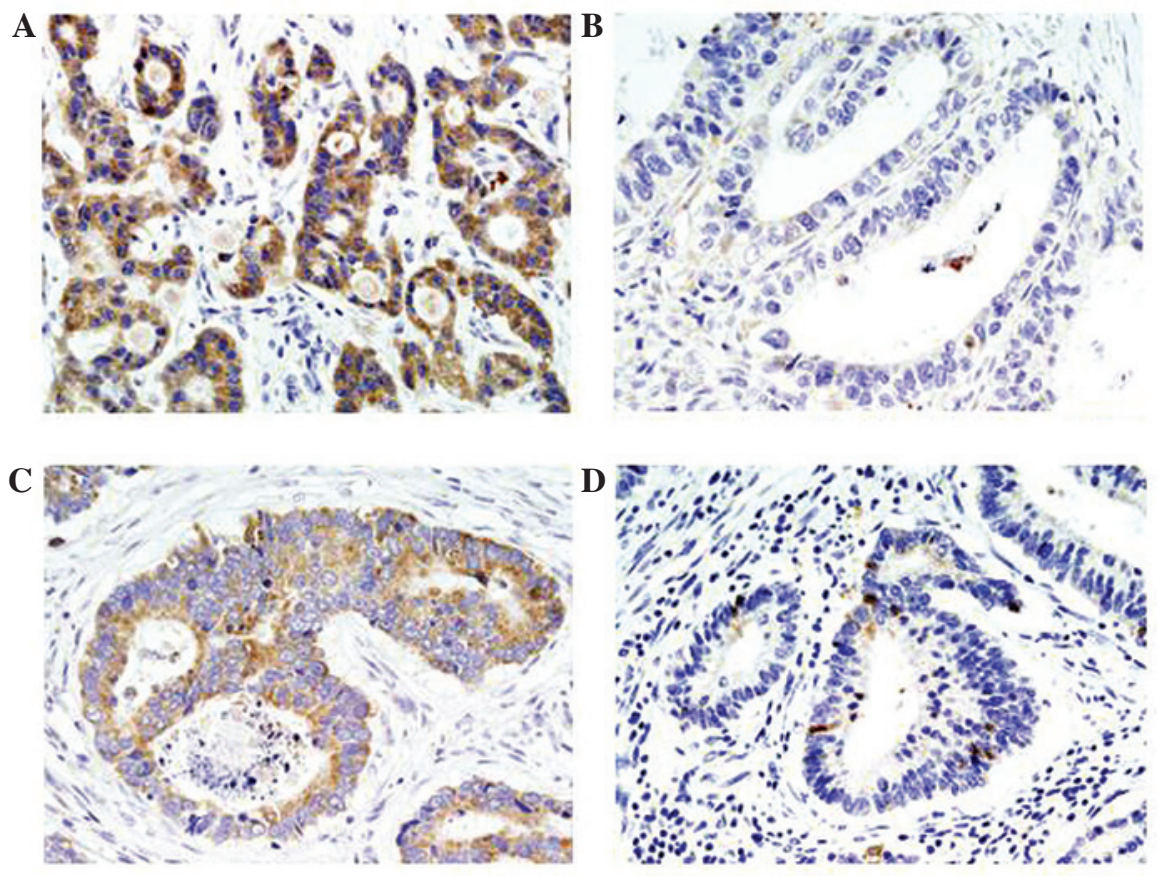

Figure 1. Immunhistochemical staining of Smac/DIABLO in gastrointestinal carcinomas. Smac/DIABLO immunoreactivity was predominantly observed in the cytoplasm. (A) High expression of Smac/DIABLO in gastric carcinoma. (B) Low expression of Smac/DIABLO in gastric carcinoma. (C) High expression of Smac/DIABLO in rectal carcinoma. (D) Low expression of Smac/DIABLO in rectal carcinoma. Smac/DIABLO, second mitochondria-derived activator of caspases/direct inhibitor of apoptosis-binding protein with low pI. (A-D, magnification, x200).

parameters and the expression of Smac/DIABLO, a trend towards an association between a decreased expression level of Smac/DIABLO and tumor stage was identified.

Association between Smac/DIABLO expression in gastric and colorectal carcinomas. A correlation was identified between Smac/DIABLO and nuclear survivin $(\mathrm{r}=0.245 ; \mathrm{P}<0.01)$ in well- to moderately-differentiated colorectal adenocarcinomas. However, in all cases, the expression of Smac/DIABLO was not significantly associated with survivin expression $(\mathrm{P}=0.08)$.

\section{Discussion}

In the present study, the expression of Smac/DIABLO in gastric and colorectal carcinomas was investigated and compared by immunohistochemistry. Smac/DIABLO-positive staining was observed in 46\% (33/72) and 69\% (54/78) of gastric and colorectal carcinomas, respectively. These results revealed that the expression of Smac/DIABLO was significantly higher in colorectal carcinoma than in gastric carcinoma $(\mathrm{P}<0.01)$. Regarding the association between Smac/DIABLO expression and tumor type, Yoo et al (8) analyzed archival tissues of 100 carcinomas and 50 sarcomas from various origins using immunohistochemistry. The study reported that Smac/DIABLO was differentially expressed among cancer types and indicated that gastric, colorectal and ovarian carcinomas exhibited a high frequency of Smac/DIABLO expression, whereas Smac/DIABLO expression in prostate carcinoma and non-small cell lung carcinoma was low. According to previous studies, the positive rates of expression in gastric and colorectal carcinomas are 13-70\% and $66-90 \%$, respectively $(9,10,27-29)$. Taken together, these studies indicate that Smac/DIABLO expression in colorectal carcinoma 
Table II. Correlation between Smac/DIABLO expression and clinicopathological parameters in gastric adenocarcinomas.

\begin{tabular}{|c|c|c|c|}
\hline \multirow{2}{*}{$\begin{array}{l}\text { Clinicopathological } \\
\text { parameters }\end{array}$} & \multirow[b]{2}{*}{ Total, $\mathrm{n}$} & \multicolumn{2}{|c|}{$\begin{array}{c}\text { Smac/DIABLO } \\
\text { expression, n }(\%)\end{array}$} \\
\hline & & High & Low \\
\hline \multicolumn{4}{|l|}{ Age, years } \\
\hline$<60$ & 10 & $4(40)$ & $6(60)$ \\
\hline$>60$ & 62 & $29(47)$ & $33(53)$ \\
\hline \multicolumn{4}{|l|}{ Gender } \\
\hline Male & 51 & $23(45)$ & $28(55)$ \\
\hline Female & 21 & $10(48)$ & $11(52)$ \\
\hline \multicolumn{4}{|l|}{ Location } \\
\hline Cardia & 17 & $7(41)$ & $10(59)$ \\
\hline Fundus & 31 & $15(48)$ & $16(52)$ \\
\hline Antrum & 24 & $11(46)$ & $13(54)$ \\
\hline \multicolumn{4}{|l|}{ Depth of invasion } \\
\hline pT2 & 17 & $11(65)$ & $6(35)$ \\
\hline pT3 & 20 & $9(45)$ & $11(55)$ \\
\hline pT4 & 35 & $13(37)$ & $22(63)$ \\
\hline \multicolumn{4}{|c|}{ Lymph node metastasis } \\
\hline pNO & 23 & $14(61)$ & $9(39)$ \\
\hline $\mathrm{pN} 1$ & 15 & $4(27)$ & $11(73)$ \\
\hline $\mathrm{pN} 2$ & 11 & $4(36)$ & $7(64)$ \\
\hline $\mathrm{pN} 3$ & 23 & $11(48)$ & $12(52)$ \\
\hline \multicolumn{4}{|l|}{ Lymphatic invasion } \\
\hline Negative & 16 & $7(44)$ & $9(56)$ \\
\hline Positive & 56 & $26(46)$ & $30(54)$ \\
\hline \multicolumn{4}{|l|}{ Vascular invasion } \\
\hline Negative & 38 & $14(37)$ & $24(63)$ \\
\hline Positive & 34 & $19(56)$ & $15(44)$ \\
\hline \multicolumn{4}{|l|}{ UICC p-Stage } \\
\hline IB & 11 & $8(73)$ & $3(27)$ \\
\hline IIA and IIB & 22 & $9(41)$ & $13(59)$ \\
\hline IIIA, IIIB and IIIC & 39 & $16(41)$ & $23(59)$ \\
\hline
\end{tabular}

UICC, Union for International Cancer Control; Smac/DIABLO, second mitochondria-derived activator of caspases/direct inhibitor of apoptosis-binding protein with low $\mathrm{pI}$.

is higher than that in gastric carcinoma, however, differences may be observed due to the different counting methods used.

The results of the present study demonstrated that Smac/DIABLO expression in well- to moderately-differentiated gastric and colorectal carcinomas was higher than that in poorly-differentiated adenocarcinomas. However, no statistically significant difference was identified.Kim et al (27) reported that Smac/DIABLO expression was associated with a higher proportion of diffuse histology types than overall cases (intestinal, diffuse and mixed types). By contrast, Shibata et al (28) identified a significant correlation between Smac/DIABLO expression and tumor differentiation $(\mathrm{P}<0.0001)$, whereby patients with high Smac/DIABLO expression presented more
Table III. Correlation between Smac/DIABLO expression and clinicopathological parameters in colorectal adenocarcinomas.

\begin{tabular}{|c|c|c|c|}
\hline \multirow{2}{*}{$\begin{array}{l}\text { Clinicopathological } \\
\text { parameters }\end{array}$} & \multirow[b]{2}{*}{ Total, $\mathrm{n}$} & \multicolumn{2}{|c|}{$\begin{array}{l}\text { Smac/DIABLO } \\
\text { expression, n (\%) }\end{array}$} \\
\hline & & High & Low \\
\hline \multicolumn{4}{|l|}{ Age, years } \\
\hline$<60$ & 22 & $16(73)$ & $6(27)$ \\
\hline$>60$ & 56 & $38(68)$ & $18(32)$ \\
\hline \multicolumn{4}{|l|}{ Gender } \\
\hline Male & 44 & $29(66)$ & $15(34)$ \\
\hline Female & 34 & $25(74)$ & $9(26)$ \\
\hline \multicolumn{4}{|l|}{ Location } \\
\hline Right colon & 24 & $16(67)$ & $8(33)$ \\
\hline Left colon & 24 & $19(79)$ & $5(21)$ \\
\hline Rectum & 30 & $19(63)$ & $11(37)$ \\
\hline \multicolumn{4}{|l|}{ Depth of invasion } \\
\hline pT2 & 7 & $5(71)$ & $2(29)$ \\
\hline pT3 & 46 & $31(67)$ & $15(33)$ \\
\hline pT4 & 25 & $18(72)$ & $7(28)$ \\
\hline \multicolumn{4}{|c|}{ Lymph node metastasis } \\
\hline pNO & 35 & $25(71)$ & $10(29)$ \\
\hline pN1 & 31 & $23(74)$ & $8(26)$ \\
\hline $\mathrm{pN} 2$ & 12 & $6(50)$ & $6(50)$ \\
\hline \multicolumn{4}{|l|}{ Lymphatic invasion } \\
\hline Negative & 24 & $16(67)$ & $8(33)$ \\
\hline Positive & 54 & $38(70)$ & $16(30)$ \\
\hline \multicolumn{4}{|l|}{ Vascular invasion } \\
\hline Negative & 40 & $32(80)^{\mathrm{a}}$ & $8(20)$ \\
\hline Positive & 38 & $22(58)^{\mathrm{a}}$ & $16(42)$ \\
\hline \multicolumn{4}{|l|}{ UICC p-Stage } \\
\hline IB & 6 & $5(83)$ & $1(17)$ \\
\hline IIA and IIB & 28 & $20(71)$ & $8(29)$ \\
\hline IIIA, IIIB and IIIC & 44 & $29(66)$ & $15(34)$ \\
\hline
\end{tabular}

${ }^{\mathrm{a}} \mathrm{P}<0.05$, negative vascular invasion vs. positive vascular invasion. UICC, Union for International Cancer Control; Smac/DIABLO, second mitochondria-derived activator of caspases/direct inhibitor of apoptosis-binding protein with low $\mathrm{pI}$.

differentiated tumors. Yoo et al (8) identified no correlation between histological subtype (diffuse type vs. intestinal type) and the expression of Smac/DIABLO (8). Based on these results, we hypothesized that there is no association between the expression of Smac/DIABLO and tumor differentiation in gastric and colorectal carcinomas.

Furthermore, the association between Smac/DIABLO expression and clinicopathological parameters was investigated in the present study. In colorectal carcinomas, the level of Smac/DIABLO expression was significantly higher in the cases without vascular invasion than in the cases with vascular invasion $(\mathrm{P}<0.05)$. However, this association was not identified in gastric carcinoma. Previous studies have demonstrated that 
Smac/DIABLO expression is associated with a good prognosis and low tumor stage (8-11). In the present study, a trend towards an association between decreased Smac/DIABLO expression and pathological stage in gastric and colorectal carcinomas was observed, however, no statistically significant difference was identified. Endo et al (9) reported that no significant difference was present between the two Smac/DIABLO-positive and -negative tumor groups with respect to tumor size, tumor location, histological differentiation and lymphatic and venous invasion. The clinical significance of Smac/DIABLO expression in various cancers remains unclear. Therefore, additional comprehensive studies are required to elucidate the clinical significance of Smac/DIABLO expression in gastric and colorectal carcinomas.

In the present study, the expression of Smac/DIABLO and nuclear survivin were found to correlate in well- to moderately-differentiated colorectal adenocarcinomas $(r=0.245$; $\mathrm{P}<0.01)$. De Oliveira Lima et al (30) identified a correlation between the expression of survivin and Smac/DIABLO in colorectal carcinoma using immunohistochemistry. The results of the present study only identified a correlation in well- to moderately-differentiated cases, however, the correlation was not observed in all colorectal cancer cases. By contrast, Kim et al (27) revealed that Smac/DIABLO expression was not associated with survivin, whereas Smac/DIABLO expression was found to inversely correlate with the expression of XIAP, an IAP, using immunohistochemistry. To the best of our knowledge, only a small number of studies have demonstrated the association between Smac/DIABLO and survivin expression using immunohistochemistry. Endo et al (9) proposed that the decrease of Smac/DIABLO expression is an independent factor of poor prognosis for colorectal cancer patients, while other studies have indicated that survivin may be a marker for tumor progression and prognosis $(5,31,32)$. Survivin is found in the nucleus and cytoplasm. Nuclear survivin is considered a promoter of cell proliferation, whereas cytoplasmic survivin is considered to exhibit cytoprotective effects $(16,33)$, however, the mechanisms involved remain unclear. Previous studies have indicated that nuclear and cytoplasmic survivin expression is associated with an improved prognosis in gastric carcinomas. By contrast, in colorectal carcinoma, the upregulation of cytoplasmic survivin is associated with a poor prognosis (31,32,34-37). However, there is no disagreement between the results of previous studies and the present study. At present, the association between survivin or Smac/DIABLO and the clinical prognosis remains controversial. Thus, further studies are required to confirm the association between Smac/DIABLO expression and nuclear or cytoplasmic survivin expression.

In conclusion, the present study demonstrated that the expression of Smac/DIABLO was significantly higher in colorectal carcinoma than in gastric carcinoma. Additionally, a correlation was found between the expression of Smac/DIABLO and nuclear survivin in well- to moderately-differentiated colorectal adenocarcinomas $(\mathrm{r}=0.245 ; \mathrm{P}<0.01)$. Based on these results, we hypothesized that gastric and colorectal carcinomas differ in their levels of Smac/DIABLO expression. These results, in addition to our previous results, indicate that not only LC3 and survivin expression levels, but also Smac/DIABLO expression levels, are significantly higher in colorectal carcinoma than in gastric carcinoma $(24,25)$. We hypothesized that the expression of Smac/DIABLO in colorectal carcinoma may be upregulated to suppress the anti-apoptotic effect of survivin. Furthermore, the results of this study indicate that the analysis of Smac/DIABLO, survivin and LC3 expression in colorectal carcinoma is likely to aid cancer therapy due to the involvement of these markers in apoptosis and/or autophagy.

\section{Acknowledgements}

The authors would like to thank Dr Masayuki Shintaku and Dr Toshihiko Miyake for their kind support. This study was supported by a Grant-in-Aid for Scientific Research (grant no. 23590396) from the Japan Society for the Promotion of Science.

\section{References}

1. Shiozaki EN and Shi Y: Caspases, IAPs and Smac/DIABLO: mechanisms from structural biology. Trends Biochem Sci 29: 486-494, 2004.

2. Cheung HH, LaCasse EC and Korneluk RG: X-linked inhibitor of apoptosis antagonism: strategies in cancer treatment. Clin Cancer Res 12: 3238-3242, 2006.

3. Srinivasula SM, Hegde R, Saleh A, et al: A conserved XIAP-interaction motif in caspase-9 and Smac/DIABLO regulates caspase activity and apoptosis. Nature 410: 112-116, 2001.

4. Song Z, Yao X and Wu M: Direct interaction between survivin and Smac/DIABLO is essential for the anti-apoptotic activity of survivin during taxol-induced apoptosis. J Biol Chem 278: 23130-23140, 2003

5. Miura K, Fujibuchi W, Ishida K, et al: Inhibitor of apoptosis protein family as diagnostic markers and therapeutic targets of colorectal cancer. Surg Today 41: 175-182, 2011.

6. Grzybowska-Izydorczyk O, Cebula B, Robak T and Smolewski P: Expression and prognostic significance of the inhibitor of apoptosis protein (IAP) family and its antagonists in chronic lymphocytic leukaemia. Eur J Cancer 46: 800-810, 2010.

7. Wrzesień-Kuś A, Smolewski P, Sobczak-Pluta A, Wierzbowska A and Robak T: The inhibitor of apoptosis protein family and its antagonists in acute leukemias. Apoptosis 9: 705-715, 2004.

8. Yoo NJ, Kim HS and Kim SY, et al: Immunohistochemical analysis of Smac/DIABLO expression in human carcinomas and sarcomas. APMIS 111: 382-388, 2003.

9. Endo K, Kohnoe S, Watanabe A, et al: Clinical significance of Smac/DIABLO expression in colorectal cancer. Oncol Rep 21: 351-355, 2009.

10. Yan H, Yu J, Wang R, Jiang S, Zhu K, Mu D and Xu Z: Prognostic value of Smac expression in rectal cancer patients treated with neoadjuvant therapy. Med Oncol 29: 168-173, 2012.

11. Zheng LD, Tong QS, Wang L, Liu J and Qian W: Stable transfection of extrinsic Smac gene enhances apoptosis-inducing effects of chemotherapeutic drugs on gastric cancer cells. World J Gastroenterol 11: 79-83, 2005.

12. Li F: Survivin study: what is the next wave? J Cell Physiol 197: 8-29, 2003.

13. Ambrosini G, Adida C and Altieri DC: A novel anti-apoptosis gene, survivin, expressed in cancer and lymphoma. Nat Med 3: 917-921, 1997.

14. Liston P, Fong WG and Korneluk RG: The inhibitors of apoptosis: there is more to life than Bcl2. Oncogene 22: 8568-8580, 2003

15. Li F, Ackermann EJ, Bennett CF, et al: Pleiotropic cell-division defects and apoptosis induced by interference with survivin function. Nat Cell Biol 1: 461-466, 1999.

16. Knauer SK, Mann W and Stauber RH: Survivin's dual role: an export's view. Cell Cycle 6: 518-521, 2007.

17. Sah NK, Khan Z, Khan GJ and Bisen PS: Structural, functional and therapeutic biology of survivin. Cancer Lett 244: 164-171, 2006.

18. Andersen MH, Svane IM, Becker JC and Straten PT: The universal character of the tumor-associated antigen survivin. Clin Cancer Res 13: 5991-5994, 2007. 
19. Choi J and Chang H: The expression of MAGE and SSX, and correlation of COX2, VEGF, and survivin in colorectal cancer. Anticancer Res 32: 559-564, 2012.

20. Wang TT, Qian XP and Liu BR: Survivin: potential role in diagnosis, prognosis and targeted therapy of gastric cancer. World J Gastroenterol 13: 2784-2790, 2007.

21. Kerr JF, Winterford CM and Harmon BV: Apoptosis. Its significance in cancer and cancer therapy. Cancer 73: 2013-2026, 1994.

22. Reed JC: Mechanisms of apoptosis. Am J Pathol 157: 1415-1430, 2000.

23. Lavrik IN, Golks A and Krammer PH: Caspases: pharmacological manipulation of cell death. J Clin Invest 115: 2665-2672, 2005.

24. Shintani M, Sangawa A, Yamao N, Miyake T and Kamoshida S: Immunohistochemical analysis of cell death pathways in gastrointestinal adenocarcinoma. Biomed Res 32: 379-386, 2011.

25. Shintani M, Sangawa A, Yamao N and Kamoshida $S$ : Immunohistochemical expression of nuclear and cytoplasmic survivin in gastrointestinal carcinoma. Int J Clin Exp Pathol 6 : 2919-2927, 2013

26. Sobin LH, Gospodarowicz MK and Wittekind C (eds): TNM Classification of Malignant Tumours. 7th edition, Wiley-Blackwell, New Jersey, 2009.

27. Kim MA, Lee HE, Lee HS, Yang HK and Kim WH: Expression of apoptosis-related proteins and its clinical implication in surgically resected gastric carcinoma. Virchows Arch 459: 503-510, 2011

28. Shibata T, Noguchi T, Takeno S, Gabbert HE, Ramp U and Kawahara K: Disturbed XIAP and XAF1 expression balance is an independent prognostic factor in gastric adenocarcinomas. Ann Surg Oncol 15: 3579-3587, 2008.

29. DA Silva LC, Forones NM, Ribeiro DA, Ihara SS, Gomes TS Neto RA and Oshima CT: Immunoexpression of DIABLO, AIF and cytochrome $\mathrm{C}$ in gastric adenocarcinoma assessed by tissue Microarray. Anticancer Res 33: 647-653, 2013.
30. De Oliveira Lima F, De Oliveira Costa H, Barrezueta LF, et al: Immunoexpression of inhibitors of apoptosis proteins and their antagonist SMAC/DIABLO in colorectal carcinoma: correlation with apoptotic index, cellular proliferation and prognosis. Oncol Rep 22: 295-303, 2009.

31. Fang YJ, Lu ZH Wang GQ, et al: Elevated expressions of MMP7, TROP2, and survivin are associated with survival, disease recurrence, and liver metastasis of colon cancer. Int J Colorectal Dis 24: 875-884, 2009.

32. Okada E, Murai Y, Matsui K, Isizawa S, Cheng C, Masuda $\mathrm{M}$ and Takano Y: Survivin expression in tumor cell nuclei is predictive of a favorable prognosis in gastric cancer patients. Cancer Lett 163: 109-116, 2001

33. Stauber RH, Mann W and Knauer SK: Nuclear and cytoplasmic survivin: molecular mechanism, prognostic, and therapeutic potential. Cancer Res 67: 5999-6002, 2007.

34. Ponnelle T, Chapusot C, Martin L, et al: Cellular localisation of survivin: impact on the prognosis in colorectal cancer. J Cancer Res Clin Oncol 131: 504-510, 2005.

35. Qi G, Tuncel H, Aoki E, et al: Intracellular localization of survivin determines biological behavior in colorectal cancer. Oncol Rep 22: 557-562, 2009.

36. Lee YY, Yu CP, Lin CK, Nieh S, Hsu KF, Chiang H and Jin JS Expression of survivin and cortactin in colorectal adenocarcinoma: association with clinicopathological parameters. Dis Markers 26: 9-18, 2009.

37. Vallböhmer D, Drebber U, Schneider PM, et al: Survivin expression in gastric cancer: Association with histomorphological response to neoadjuvant therapy and prognosis. J Surg Oncol 99: 409-413, 2009. 